Internal Report

DESY $M-80 / 17$

August 1980

A. Febel and G. Hemmie 
DESY behält sich alle Rechte für den Fall der Schutzrechtserteilung und für die wirtschaftliche Verwertung der in diesem Bericht enthaltenen Informationen vor.

DESY reserves all rights for commercial use of information included in this report, especially in case of apply for or grant of patents.

"DIE VERANTHORTUNG FUR DEN INHALT

DIESES INTERNEN BERICHTES LIEGT

AUSSCHLIESSLICH BEIM VERFASSER." 


\title{
STATUS AND PERFORMANCE OF PIA
}

A. Febel and G. Hemmie

Deutsches Elektronen-Synchrotron DESY, Hamburg, W. Germany

\begin{abstract}
The storage ring PIA, designed to produce intensive single $500 \mathrm{MHz}$ positron bunches, was installed last year. In June positrons were accumulated and compressed in a single bunch of $l$ ns length for the first time. The accumulation efficiency was raised up to $56 \%$ by matching the linac beam to the PIA optics and by compensating the sextupol components in the bending magnets by additional sextupoles. Since July 1979 PIA has been running routinely for PETRA and DORIS:

The linac ( $50 \mathrm{~Hz}, 450 \mathrm{MeV}$ ) delivers 9 bursts ( $100 \mathrm{~ns}$, $2 \times 109$ positrons each), which are accumulated and damped to a bunch length of $80 \mathrm{~cm}$ by a $10.4 \mathrm{MHz}$ cavity. Then the bunch is compressed to $25 \mathrm{~cm}$ length by a $125 \mathrm{MHz}$ if system in $60 \mathrm{~ms}$ and then ejected.

Up to 1010 positrons were accumulated in a circulating bunch. The ejection efficiency is $90 \%$ the repetition rate of PIA $4 \mathrm{~Hz}$.
\end{abstract}

\section{INTRODUCTION}

As part of the PETRA injection system the small storage ring PIA has the task to produce single 2 ns positron bunches of high intensity. This is necessary for the following reasons: The design number of positrons in each of four PETRA buckets is $1 \times 1012$ (these values are meanuhile achieved). Since the damping time for horizontal betatron oscillations in PETRA is about $100 \mathrm{~ms}$ at $7 \mathrm{GeV}$ at least $5 \times 10^{8}$ positrons have to be delivered to the PETRA ring within a single bunch, resulting in a filling time of 15 min if there were no losses. The DESY positron linac yields only $2 \times 10^{7}$ particles within $2 \mathrm{~ns}$. One possible method to improve the intensity in the $500 \mathrm{MHz}$ bunches is the use of an intermediate storage ring. So in the first year of PETRA operation the DORIS storage ring was run in this mode as part of the PETRA injection system ${ }^{1}$ ). This system worked fine inspite of its complexity, but DORIS was almost fully occupied by the PETRA injection and could not be used for high energy physics. Therefore in $1978 / 79$ the old idea of having a small storage ring at the end of the linac was realised. Due to PIA the PETRA injection system ls now very simple (Fig. 1): Single positron bunches of high intensity created in PIA are injected at 450 MeV into the DESY synchrotron, accelerated up to 7 GeV, transferred to PETRA and accumulated there in the assigned buckets. The repetition rate is $4 \mathrm{~Hz}$, comfortably small as compared to the damping rate in PETRA.

WORKING PRINCIPLE OF PIA

PIA works in the following way: The linac, running at $50 \mathrm{~Hz}$, delivers 9 bursts of positron S-band bunches with a pulse length of $2100 \mathrm{~ns}$ each and with $2 \times 10^{9}$ particles within an energy spread of $\pm .5 \%$. They are transferred to PIA via a small transport system, injected and accumulated there. 


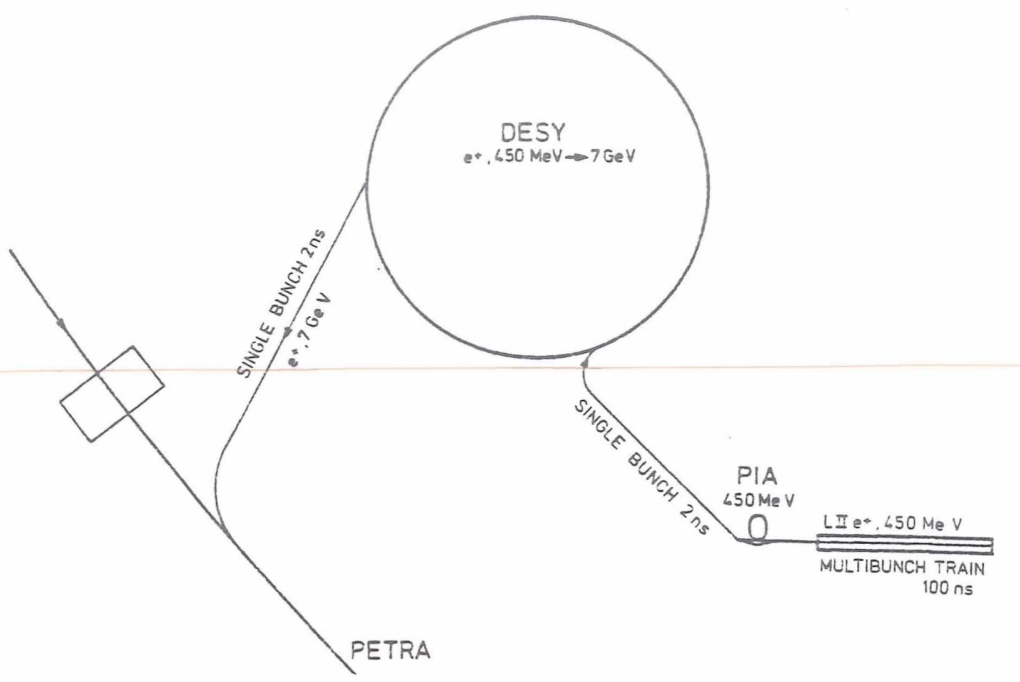

Fig. 1: PETRA Fositron Irijection

The energy of the positrons is 450 MeV. PIA has a circumference of $28.8 \mathrm{~m}$ ( = 1/80 of PETRA's), the revolution time is 96 ns. The beam is injected within one turn with the help of two beam bump kickers which have a horizontal betation phase shift of $180^{\circ}$ between them and are respectively located upstream and dounstream of an injection septum magnet. These kickers move the already accumulated beam every 20 ms to the edge of the current strip of the septum before the next linac pulse is injected.

Since the bending radius of the PIA magnets is $1 \mathrm{~m}$, the energy loss of the $450 \mathrm{MeV}$ beam is $3.63 \mathrm{keV}$ per turn. This loss is compensated by a $10.4 \mathrm{MHz}$ cavity (harmonic number $h=1$ ) that runs at a peak gap voltage of $22 \mathrm{kV} l \mathrm{lo}-$ ding to a synchronous phase angle of $9.5^{\circ}$. The radial betatron oscillations ace damped with a time constant of $24 \mathrm{~ms}$ which allows an injection repetition rate of $50 \mathrm{~Hz}$. In longitudinal direction the originally $24 \mathrm{~m}$ long burst is damped down to a bunch length of $80 \mathrm{~cm}$ with a time constant of $12 \mathrm{~ms} .20 \mathrm{~ms}$ after the last ( $i . e$. the 9 th) injection a second cavity with a resonant frequency of $125 \mathrm{MHz}(\mathrm{h}=12)$ is powered. It's peak voltage of 21 keV compresscs the bunch adiabatically within $100 \mu s$ and then the bunch length is damped down aqain by radiation to $2 \sigma=25 \mathrm{~cm}$ within a time of $40 \mathrm{~ms}$. This bunch fits well into the $500 \mathrm{MHz}$ buckets of the DESY synchrotron. It will be ejected using an cjection kicker magnet and the same septum magnet that is used for injection (Fig. 2).

\section{SOME COMPONENTS IN DETAIL}

There are 8 bending magnets, $45^{\circ}$ sector magnets with a magnetic field of $1.43 \mathrm{~T}$ at $450 \mathrm{MeV}$ on the central orbit and with superimposed defocusing gradient field of $.75 \mathrm{~T} / \mathrm{m}\left(\mathrm{k}=.5 \mathrm{~m}^{-2}\right)$. Measurements shoved that at this cxcitation the mannets are already partially saturated and contain sextupole components which cannot be neglected. 4 horizontally focusing (QF) and 4 de- 


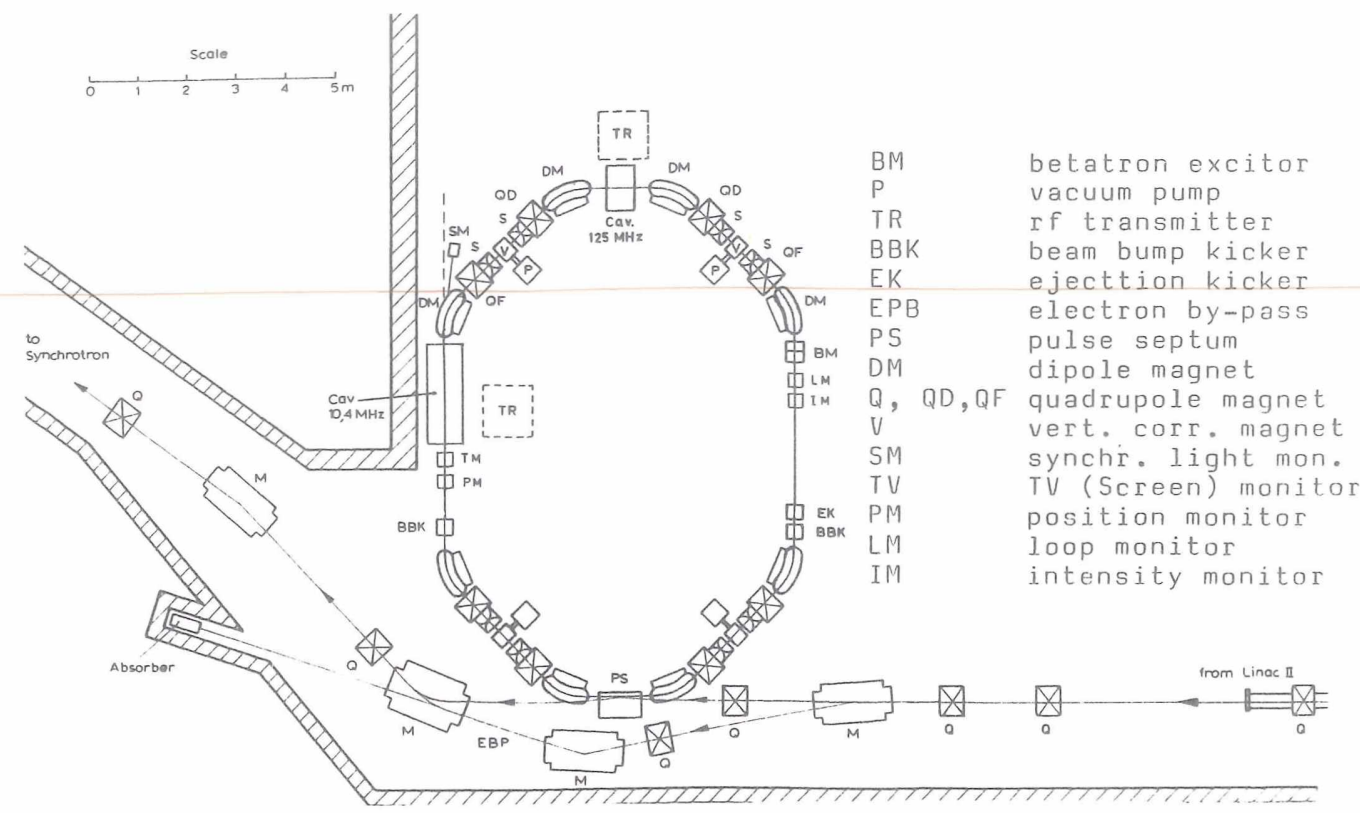

Fig. 2: The Storage Ring PIA

focusing (QD) quadrupole magnets with focusing lengths in the range of 1.5 to $2 \mathrm{~m}$ determine the working points to be around 1.6 to 1.8 . Without the quads PIA would be a weak focusing machine. $2 \times 4$ sextupole magnets of the PETRA type are installed, two of them each between two adjacent quads. They were thought to be necessary to compensate the chromaticity of the machine to zero or to positive values in order to overcome the head-tail instability. At present four of them are used to raise the acceptance of PIA which would otherwise be heavily reduced by the sextupole fields in the bending magnets.

6 short dipole magnets in the straight sections allow for corsecting the closed orbit vertically. Corrections in the horizontal plane are achieved by powering backleg windings in the PIA bending magnets.

A pressure of less than $3.5 \times 10^{-9}$ torr in the vacuum system is provided by nine $400 \mathrm{l} / \mathrm{s}$ pumps beneath the bigger tanks (kickers, septum and cavities) and four $30 \mathrm{l} / \mathrm{s}$ pumps in the short straight sections, leading to a lifetime of nearly an hour which is mainly determined by Coulomb scattering at the nuclei of the restgas. This is by far sufficient for normal operation of the storage ring and is even good enough for measurements with the stored beam.

Both cavities are made of aluminium. The $10.4 \mathrm{MHz}$ one has an unloaded $Q_{0}$ of 3000 and a shunt impedance of $43 \mathrm{k} \Omega$. It is driven by a $7 \mathrm{~kW}$ tetrode transmitter. In order to avoid self-bunching of the beam, the Q-value of the $125 \mathrm{MHz}$ cavity is reduced to 500 during the injection pulses by a loop coupled to an $\mathrm{rf}$ load. Just before being excited the resonator is tuned into resonance by switching on a short circuit a quarter wavelength apart from 
the loop. The input power is $200 \mathrm{~W}$.

OPERATION AND PERFORMANCE OF PIA

The PIA ring was designed in the years $1977 / 78$ and assembled in February 1979 with all components except the rf systems. Soon after the first injection 500 beam revolutions could be seen in PIA. More were not expected at that time due to the radiation energy loss of the positron beam. The If cavities and the transmitters were installed in May 1979 and in June the first accumulated bunch circulated in PIA. It had already the short length to fit into a single 500 MHz bucket of DESY and PETRA (see Fig. 3).

The initially very low accumulation rate raised by matching the linac positron beam to the acceptance of PIA and by compensating the inherent sextupole components in the dipole fields. The last cure was found by using the LIMATRA tracking program. The compensation was dorie by powering one family of four sextupole magnets and shifting the chromaticity $\xi=\frac{\Delta Q}{\Delta p / p}$ from $\xi_{x}=-16$ and $\xi_{z}=+5$ back to the natural values of -4 and -1.3 as calculated from linear optics. There was no necessity for totally compensating the chromaticity since at circulating currents of less than $20 \mathrm{~mA}$ no single bunch instability could be ovserved.

With these two procedures the accumulation rate was brought up to $90 \mathrm{~mA} / \mathrm{s}$. The accumulation efficiency is $56 \%$ and $90 \%$ of the circulating beam (up to $10^{10}$ particles) are ejected and transferred to DESY and PETRA. A typical scope picture showing the accumulation process in PIA during ore cycle is seen in Fig. 4 .

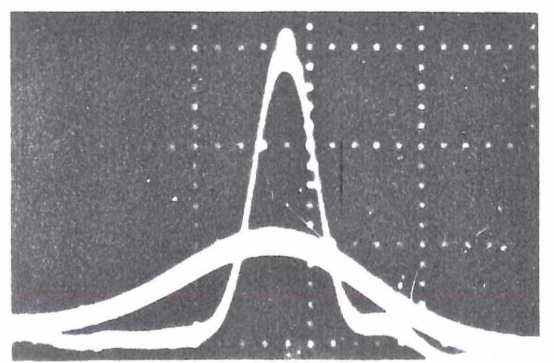

Fig. 3: Bunch Length before and after Compression by the $125 \mathrm{MHz}$ if System (Time Scale: 2 ns/div)

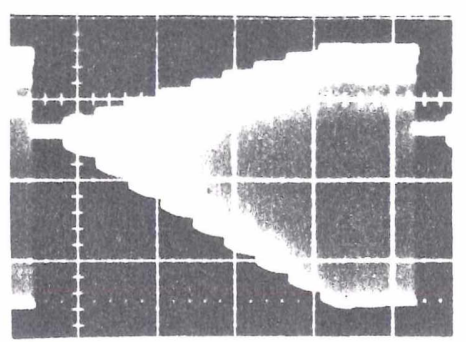

Fig. 4: Positron Current in PIA (Time Scale: $50 \mathrm{~ms} / \mathrm{div}$ )

PIA is now operated with nearly the same optics that had been proposed 2,3 ) with almost zero dispersion at the injection point, large horizontal accoptance and a relatively large dispersion in the long straight sections. The measured $Q$-values are $Q_{x}=1.64$ and $Q_{z}=1.61$. The ratio of the beam height to the bean width was measured by the synchrotron light monitor to be about 5 iv, but it indeed rises to $100 \%$ when shifting the working point to $Q_{x}=0_{z}$. 


\section{REFERENCES}

1) A.Febel, G. Hemrie, G. Mühlhaupt, K. Wille "PETRA INJECTION SYSTEM", Proceedings of the Xth International Conference on High Energy Accelerators, Protvino, July 1977, Vol 1, P. 464

2) A. Febel, G. Hemmie, G. Mühlhaupt "Auslegung des Zwischenspeichers für die Positroneninjektion nach PETRA", Technical Note DESY-PET 77/29

3) A. Febel, G. Hemmie, "PIA, the Positron Intensity Accumulator for the PETRA Injection", IEEE Trans., June 1979, Vol. NS-26, No. 3, p. 3244 